\title{
A TOPOLOGICAL METHOD FOR BOUNDED SOLUTIONS OF NONAUTONOMOUS ORDINARY DIFFERENTIAL EQUATIONS
}

\author{
JAMES R. WARD, JR.
}

\begin{abstract}
The existence of bounded solutions to nonlinear nonautonomous ordinary differential equations is studied. This is done by associating the given equation to nonlinear autonomous ones by means of a family of skew-product flows related by homotopy. The existence of a bounded solution to the original differential equation is then related to the nontriviality of a certain Conley index associated with the autonomous differential equations. The existence of nontrivial bounded solutions is also considered. The differential equations studied are perturbations of homogeneous ones.
\end{abstract}

The purpose of this paper is the further development and application of an approach introduced in [W] for the study of nonautonomous ordinary differential equations. The key idea is to apply the index theory of Conley [C] to compact isolated invariant sets in the skew product flows associated with such equations, and to then form conclusions concerning the existence, multiplicity, and stability properties of bounded solutions of the original differential equation.

Many ordinary differential equations in $\mathbb{R}^{N}$ may be viewed as perturbations of homogeneous problems, and these are the type of problems this paper is devoted to. We will thus consider ordinary differential equations which may be written in the form

$$
x^{\prime}=f(x, t)+g(x, t), \quad\left(x^{\prime}=d x / d t\right)
$$

where $f(\lambda x, t)=\lambda^{p} f(x, t)$, for all $\lambda>0$ and some $p>1$, and $g(x, t)$ is of lower order in $x$ at infinity. We usually assume $f$ and $g$ are uniformly almost periodic in $t$.

Our interest in using the Conley index theory lies in the fact that the index is, under suitable conditions, a homotopy invariant which carries both existence and stability information on compact isolated invariant sets. We introduce skew product flows into the problem because the index is defined only for (local) dynamical systems, so that time invariance is essential.

In order to study $(0.1)$ we introduce a skew product flow associated with $(0.1)$ on the product space $\mathbb{R}^{N} \times H(f) \times H(g)$ where $H(f)$ denotes the hull of the

Received by the editors July 13, 1989 and, in revised form, July 30, 1990.

1980 Mathematics Subject Classification (1985 Revision). Primary 34A34, 34C11. Secondary $34 \mathrm{C} 27,34 \mathrm{C} 35$.

Key words and phrases. Nonlinear, nonautonomous ordinary differential equations, almostperiodic solutions, bounded solutions, Conley index, skew-product flows.

Research supported in part by NSF grant RII-8996152. 
function $t \mapsto f(x, t)$. We then imbed this flow, $\pi$, into a homotopy of flows with the idea of showing $\pi$ to be equivalent on a given isolating neighborhood to a simpler flow on $\mathbb{R}^{N} \times H(f) \times H(g)$. The approach sometimes taken is to homotopy $\pi$ to a product flow where one factor of the product is defined on $\mathbb{R}^{N}$ by an autonomous ordinary differential equation and the other factor is defined in a simple way on $H(f) \times H(g)$. The index of the product flow is then calculated with the smash product of the indices of the factors. This approach raises the question of which skew-product flows may be homotopically equivalent on compact isolating neighborhoods to product flows, an interesting question we will not answer here. Related results appear in [Me-W] and [W.1].

In this paper we will relate the flow defined by $(0.1)$ to the flows defined by

$$
x^{\prime}=f^{*}(x, 0)
$$

where $f^{*} \in H(f)$. That is, we "freeze" coefficients, an idea used also by Muhamadiev [M] in connection with a degree theoretic approach to obtain existence for periodic solutions of $(0.2)$; these results are presented in [K-Z].

In $\S 1$ we prove a lemma regarding index products of the type occurring in this paper; in $\S 2$ we state some of our main results; $\S 3$ concerns linearization and nontrivial solutions; and $\S 4$ has two examples.

The reader is assumed conversant with the Conley index theory which is described in $[\mathrm{C}]$ (or see $[\mathrm{R}]$ or $[\mathrm{Sm}]$ ), with skew product flows (see, e.g. [Se]), and with uniformly almost periodic functions (see, e.g., $[\mathrm{F}])$.

\section{A LEMMA ON THE INDEX OF PRODUCTS}

Let $\pi_{1}$ and $\pi_{2}$ be (local) flows defined on locally compact metric spaces $X_{1}$ and $X_{2}$, respectively. If $K_{i} \subset X_{i}$ for $i=1,2$ are compact isolated invariant sets then $K_{1} \times K_{2}$ is a compact isolated invariant set for the product flow $\pi_{1} \times \pi_{2}$ on $X_{1} \times X_{2}$ and the Conley index of $K_{1} \times K_{2}$ in the flow $\pi_{1} \times \pi_{2}$, which we denote by $h\left(\pi_{1} \times \pi_{2}, K_{1} \times K_{2}\right)$, is equal to the smash product $h\left(\pi_{1}, K_{1}\right) \wedge$ $h\left(\pi_{2}, K_{2}\right)$. That is, the (Conley) index of a product is the smash product of the indices of the factors; see [C] or [R]. The following lemma from [Me-W] gives a sufficient condition for the index of a product to be nonzero. It is useful to us because many of our invariant sets will be product sets with the properties of those in the lemma. The proof is included for the convenience of the reader.

Lemma 1.1. Let $\pi_{i}, X_{i}, i=1,2$, be as above and suppose $X_{2}$ is a compact space. Let $K_{1} \subset X_{1}$ be a compact isolated invariant set for $\pi_{1}$, so that $K_{1} \times X_{2}$ is a compact invariant set for $\pi_{1} \times \pi_{2}$. If $h\left(\pi_{1}, K_{1}\right) \neq \overline{0}$ then $h\left(\pi_{1} \times \pi_{2}, K_{1} \times X_{2}\right)$ $\neq \overline{0}$.

Remark 1.1. Here and throughout this paper we will use the notation $\left[Y^{*}, z\right]$ to denote the homotopy type of a compact pointed space of the form $Y^{*}=$ $Y \bigcup^{\prime}\{z\}$ where $Y$ is compact $z \notin Y$ is the distinguished point, and ${ }^{\prime}$ denotes disjoint union. Thus $\left[Y^{*}, z\right]$ will always be a disconnected homotopy type. The notation $(Y, z)$ will be used for compact pointed space $Y$ with distinguished point $z$, and $[Y, z]$ for the homotopy type of $(Y, z)$. We will also generally use $A \sim B$ between pointed spaces $A, B$ to denote homotopic equivalence.

Proof of Lemma 1.1. Since $h\left(\pi_{1} \times \pi_{2}, K_{1} \times X_{2}\right)=h\left(\pi_{1}, K_{1}\right) \wedge h\left(\pi_{2}, X_{2}\right)$ and $h\left(\pi_{2}, X_{2}\right)=\left[X_{2}^{*}, z\right]$, if the lemma is false then we must have $h\left(\pi_{1}, K_{1}\right) \wedge$ $\left[X_{2}^{*}, z\right]=\overline{0}$. 
If $(A, p)$ is any representative in $h\left(\pi_{1}, K_{1}\right)$ then $h\left(\pi_{1} \times \pi_{2}, K_{1} \times X_{2}\right)=$ $\left[W, w_{0}\right]$ where $W=A \times X_{2}^{*} /\left(A \times\{z\} \cup\{p\} \times X_{2}^{*}\right), w_{0}=\left[A \times\{z\} \cup\{p\} \times X_{2}^{*}\right]$, and $X_{2}^{*}=X_{2} \bigcup^{\prime}\{z\}$. We have obviously

$$
\begin{aligned}
\left(W, w_{0}\right) & =\left(\left(A \times\left(X_{2} \cup z\right)\right) /\left(A \times\{z\} \cup\{p\} \times\left(X_{2} \cup\{z\}\right)\right), w_{0}\right) \\
& \sim\left(\left(A \times X_{2}\right) /\left(\{p\} \times X_{2}\right), w^{*}\right)
\end{aligned}
$$

where $w^{*}=\left[\{p\} \times X_{2}\right]$.

Now suppose $\left[W, w_{0}\right]=\overline{0}$. Then, letting $W_{1}=\left(A \times X_{2}\right) /\left(\{p\} \times X_{2}\right)$, we have $\left[W_{1}, w^{*}\right]=\overline{0}$ and hence there is a homotopy $G_{\mu}, 0 \leq \mu \leq 1$, on $\left(W_{1}, w^{*}\right)$, with $G_{1}=1$, the identity, and $G_{0}(z)=w^{*}$ for all $z \in W_{1}$. Let $P:\left(W_{1}, w^{*}\right) \rightarrow$ $A$ be defined by $P(a, b)=a$ if $(a, b) \neq w^{*}$ (i.e., $\left.(a, b) \notin\{p\} \times X_{2}\right)$ and $P\left(w^{*}\right)=p$; then $P$ is a continuous mapping with the quotient topology on $\left(W_{1}, w^{*}\right)$ into $(A, p)$. Now fix $b^{*} \in X_{2}$ and define $Q: A \rightarrow\left(W, w^{*}\right)$ by $Q(a)=\left(a, b^{*}\right)$ if $a \neq p$ and $Q(p)=w^{*} ; Q$ is continuous. The homotopy $H_{\mu}=P \circ G_{\mu} \circ Q$ on $(A, p)$ shows $(A, p) \sim \overline{0}$, contrary to our hypothesis. This proves the lemma.

\section{Perturbed homogeneous PROBLEMS AND FREeZING COEFFICIENTS}

Let $N \geq 1$ and $f, g: \mathbb{R}^{N} \times \mathbb{R} \rightarrow \mathbb{R}^{N}$ continuous functions. It will always be assumed that both $f$ and $g$ are bounded and uniformly continuous on all sets of the form $K \times \mathbb{R}$ where $K$ is a compact subset of $\mathbb{R}^{N}$. It will also be assumed that both $f$ and $g$ are locally Lipschitz in their first variables, uniformly in their second variables. That is, for each $x \in \mathbb{R}^{N}$ there is a number $\delta>0$ and a number $L \geq 0$ such that $|f(x, t)-f(y, t)| \leq L|x-y|$ for all $|x-y| \leq \delta$ and $t \in \mathbb{R}$, and similarly for $g$.

For $\tau \in \mathbb{R}$ and $w: \mathbb{R}^{N} \times \mathbb{R} \rightarrow \mathbb{R}^{N}$ we let $w_{\tau}(x, t)=w(x, \tau+t)$ for $(x, t) \in \mathbb{R}^{N} \times \mathbb{R}$. The hull of $w, H(w)$, is defined by

$$
H(w)=\operatorname{cl}\left\{w_{\tau}: \tau \in \mathbb{R}\right\}
$$

where the closure is taken in the topology of uniform convergence on compact subsets of $\mathbb{R}^{N} \times \mathbb{R}$. The space of continuous functions on $\mathbb{R}^{k} \times \mathbb{R}$ into $\mathbb{R}^{n}$ with the topology of uniform convergence on compact sets will be denoted by $C_{c}\left(\mathbb{R}^{k} \times \mathbb{R}, \mathbb{R}^{n}\right)$, or just $C_{c}$ if there is no chance of confusion. The space $C_{c}$ is metrizable. The assumptions made above concerning $f$ and $g$ imply that $H(f), H(g)$, are compact in $C_{c}\left(\mathbb{R}^{N} \times \mathbb{R}, \mathbb{R}^{N}\right)$ and $H(f, g)$ is compact in $C_{c}\left(R^{N} \times \mathbb{R}, \mathbb{R}^{2 N}\right)$. Let $[f, g]$ be the mapping $(x, t) \mapsto(f(x, t), g(x, t))$ from $\mathbb{R}^{N} \times \mathbb{R}$ into $\mathbb{R}^{N} \times \mathbb{R}^{N}$. If $h^{*} \in H([f, g])$ then there are unique $f^{*} \in H(f)$ and $g^{*} \in H(g)$ with $h^{*}(x, t)=\left(f^{*}(x, t), g^{*}(x, t)\right)$ for all $(x, t) \in \mathbb{R}^{N} \times \mathbb{R}$. The natural injection $H([f, g]) \mapsto H(f) \times H(g)$ given by $h^{*} \mapsto\left(f^{*}, g^{*}\right)$ allows us to identify $h^{*}$ with $\left(f^{*}, g^{*}\right)$. That is, $[f, g]$ with $(f, g)$ so that we may think of $H([f, g])$ as a subset of $H(f) \times H(g)$, and we will write $H(f, g)$ for $H([f, g])$. In general $H(f, g)$ is a proper subset of $H(f) \times H(g)$ but sometimes these sets are equal, and this will be important in the sequel.

We are interested in the existence of bounded solutions (and associated indices) to perturbed problems such as

$$
x^{\prime}=f(x, t)+g(x, t)
$$


and

$$
x^{\prime}=f^{*}(x, t)+g^{*}(x, t), \quad\left(f^{*}, g^{*}\right) \in H(f) \times H(g) .
$$

We will do this by relating (2.1) (and $(2.1)^{*}$ ) not just to the nonautonomous system $x^{\prime}=f(x, t)$ but by relating $(2.1)$ to the family of autonomous equations

$$
x^{\prime}=f^{*}(x, 0), \quad f^{*} \in H(f) .
$$

Note that $f^{*}(\cdot, 0)$ is a function in the closure of the set $\left\{g \in C_{c}\left(\mathbb{R}^{N}, \mathbb{R}^{N}\right)\right.$ : $g=f_{\tau}(\cdot, 0)$ for some $\left.\tau \in \mathbb{R}\right\}$. If $f^{*} \in H(f)$ is a translate of $f$ then $f^{*}(x, 0)=f\left(x, \tau^{*}\right)$ for some fixed $\tau^{*} \in \mathbb{R}$; hence we say that we are "freezing" coefficients in $x^{\prime}=f(x, t)$ to obtain (2.2). Define a (local) flow $\beta$ on $\mathbb{R}^{N} \times H(f)$ by $\beta\left(v, f^{*}, t\right)=\left(x\left(t ; v, f^{*}(\cdot, 0)\right), f^{*}\right)$ where $x\left(t ; v, f^{*}(\cdot, 0)\right)$ is the solution to $(2.2)$ with $x\left(0 ; v, f^{*}(\cdot, 0)\right)=v$.

Theorem 2.1. Let $f$ and $g$ be functions as described above i.e., bounded and uniformly continuous on all sets of the form $K \times \mathbb{R}, K$ compact, and locally Lipschitz in their first variable uniformly with respect to their second variable. Assume also:

(H1) There is a number $p>1$ such that $f(\lambda x, t)=\lambda^{p} f(x, t)$ for all $\lambda>0$ and $(x, t) \in \mathbb{R}^{n} \times \mathbb{R}$.

(H2) For each $f^{*} \in H(f)$ equation (2.2) has no nonzero bounded solutions.

(H3) Let $h_{1}$ denote the Conley index of $\{0\} \times H(f)$ for the flow $\beta$; so $h_{1}=h(\beta,\{0\} \times H(f))$.

(H4) $\lim _{|x| \rightarrow \infty} g(x, t) /|x|^{p}=0$ uniformly in $t \in \mathbb{R}$.

Then if $h_{1} \neq \overline{0}$ there is $\left(f^{*}, g^{*}\right) \in H(f) \times H(g)$ such that $(2.1)^{*}$ has a solution $u^{*}=u^{*}(t)$ defined and bounded on $\mathbb{R}$.

Proof. We will divide the proof into six steps.

Step 1: Defining a homotopy of flows. Let $\left(f^{*}, g^{*}\right) \in H(f) \times H(g)$ and $\mu \in$ $[0,1]$. We will consider the family of equations

$$
x^{\prime}=f^{*}(x, \mu t)+\mu g^{*}(x, \mu t) \text {. }
$$

For $\left(\mu, f^{*}, g^{*}\right) \in[0,1] \times H(f) \times H(g)$ let $\Phi\left(\mu, f^{*}, g^{*}\right) \in C_{c}\left(\mathbb{R}^{N} \times \mathbb{R}, \mathbb{R}^{N}\right)$ be defined by

$$
\Phi\left(\mu, f^{*}, g^{*}\right)(x, t)=f^{*}(x, \mu t)+\mu g^{*}(x, \mu t) .
$$

Then $\Phi\left(\mu, f^{*}, g^{*}\right)$ is locally Lipschitz in $x$, uniformly in $t$; it is also bounded and uniformly continuous on sets of the form $K \times \mathbb{R}$ for $K$ compact in $\mathbb{R}^{N}$. We define for each $\mu \in[0,1]$ a skew-product flow on $\mathbb{R}^{N} \times H(f) \times H(g)$ as follows. For $\left(v, f^{*}, g^{*}\right) \in \mathbb{R}^{N} \times H(f) \times H(g)$ let $J\left(v, \mu, f^{*}, g^{*}\right)$ denote the maximal interval of existence for the solution to the initial value problem

$$
\begin{gathered}
x^{\prime}=\Phi\left(\mu, f^{*}, g^{*}\right)(x, t), \\
x(0)=v .
\end{gathered}
$$

We now let

$$
D(\mu)=\left\{\left(v, f^{*}, g^{*}, t\right) \in \mathbb{R}^{N} \times H(f) \times H(g) \times \mathbb{R}: t \in J\left(v, \mu, f^{*}, g^{*}\right)\right\}
$$

and define

$$
\pi_{\mu}: D(\mu) \rightarrow \mathbb{R}^{N} \times H(f) \times H(g)
$$


by

$$
\pi_{\mu}\left(v, f^{*}, g^{*}, t\right)=\left(x\left(t ; v, \mu, f^{*}, g^{*}\right), f_{\mu t}^{*}, g_{\mu t}^{*}\right) .
$$

By $x\left(t ; v, \mu, f^{*}, g^{*}\right)$ is meant the value at $t \in J\left(v, \mu, f^{*}, g^{*}\right)$ of the solution to $(2.4),(2.5)$.

At $\mu=1$ (2.6) defines the usual skew-product flow associated with (2.1), while at $\mu=0$ we "almost" have a product flow defined by "attaching" to each $\left(f^{*}, g^{*}\right) \in H(f) \times H(g)$ the autonomous flow in $\mathbb{R}^{N}$ defined by $x^{\prime}=f^{*}(x, 0)$.

Step 2: $\pi_{\mu}$ is a flow for $0 \leq \mu \leq 1$. Three conditions must hold

(i) $\pi_{\mu}\left(v, f^{*}, g^{*}, 0\right)=\left(v, f^{*}, g^{*}\right)$.

Obvious

(ii) $\pi_{\mu}\left(v, f^{*}, g^{*}, t+s\right)=\pi_{\mu}\left(\pi_{\mu}\left(v, f^{*}, g^{*}, t\right), s\right)$

for appropriate $t, s$. This is easily verified for $0 \leq \mu \leq 1$ just as in the standard $\mu=1$ case.

(iii) $\pi_{\mu}$ is continuous on $D(\mu)$.

This follows by the continuity of $\Phi$ and standard results on existence and continuity of solutions with respect to parameters.

Step 3: $\pi_{\mu}$ is continuous in $\mu \in[0,1]$. By this is meant that if

$$
\left\{\left(\mu_{n}, v_{n},\left(f_{n}^{*}, g_{n}^{*}\right), t_{n}\right): n=1,2, \ldots\right\}
$$

is a sequence in $[0,1] \times \mathbb{R}^{N} \times H(f) \times H(g) \times \mathbb{R}$ convergent to $\left(\mu, v,\left(f^{*}, g^{*}\right), t\right)$ $\in D(\mu)$ then $\left(\mu_{n}, v_{n} ;\left(f_{n}^{*}, g_{n}^{*}\right), t_{n}\right) \in D\left(\mu_{n}\right)$ for all large $n$ and

$$
\pi_{\mu_{n}}\left(v_{n}, f_{n}^{*}, g_{n}^{*}, t_{n}\right) \rightarrow \pi_{\mu}\left(v, f^{*}, g^{*}, t\right)
$$

as $n \rightarrow \infty$. Suppose we have such a sequence. Now

$$
\pi_{\mu}\left(v, f^{*}, g^{*}, t\right)=\left(x\left(t ; v, \varphi\left(\mu, f^{*}, g^{*}\right)\right), f_{t}^{*}, g_{t}^{*}\right) .
$$

By continuity of $\Phi$ we have $\Phi\left(\mu_{n}, f_{n}^{*}, g_{n}^{*}\right) \rightarrow \Phi\left(\mu, f^{*}, g^{*}\right)$ as $n \rightarrow \infty$, and by the basic theory of ordinary differential equations

$$
x\left(t_{n} ; v_{n}, \mu_{n}, f_{n}^{*}, g_{n}^{*}\right)=x\left(t_{n} ; v_{n}, \Phi\left(\mu_{n}, f_{n}^{*}, g_{n}^{*}\right)\right)
$$

is defined for all large $n$ and converges to $x\left(t ; v, \mu, \Phi\left(\mu, f^{*}, g^{*}\right)\right)$ as $n \rightarrow$ $\infty$. Since $\left(f_{n}^{*}, g_{n}^{*}\right)_{\mu_{n} t_{n}} \rightarrow\left(f^{*}, g^{*}\right)_{\mu t}=\left(f_{\mu t}^{*}, g_{\mu t}^{*}\right)$ with convergence in $C_{c}$, the continuity of $\pi_{\mu}$ follows.

Step 4: The index at $\mu=0$. At $\mu=0$ we have the flow given by

$$
\pi_{0}\left(v, f^{*}, g^{*}, t\right)=\left(x\left(t ; v, f^{*}(\cdot, 0)\right), f^{*}, g^{*}\right)
$$

where $x\left(t ; v, f^{*}(\cdot, 0)\right)$ is the solution to (2.2) with $x(0)=v$. This flow has $\{0\} \times H(f) \times H(g)$ as a compact isolated invariant set. Its index may be computed as follows. We may view the flow $\pi_{0}$ as a product of the flow $\beta$ and the flow $\tilde{\pi}\left(g^{*}, t\right)=g^{*}$. Thus we have

$$
\begin{aligned}
& h\left(\pi_{0},\{0\} \times H(f) \times H(g)\right)=h(\beta \times \tilde{\pi},\{0\} \times H(f) \times H(g)) \\
& \quad=h(\beta,\{0\} \times H(f)) \wedge h(\tilde{\pi}, H(g))=h_{1} \wedge\left[H(g)^{*}, z\right] \neq \overline{0}
\end{aligned}
$$

by Lemma 1.1 .

Step 5. The index for $0 \leq \mu \leq 1$, and existence for $(2.1)^{*}$. Suppose there were a nonempty compact set $N \subset \mathbb{R}^{N} \times H(f) \times H(g)$ with $\{0\} \times H(f) \times H(g) \subset$ 
$N$ and $N$ were an isolating neighborhood for each $\pi_{\mu}, 0 \leq \mu \leq 1$. Then if $I(\mu)$ were the largest invariant set in $N$ for the flow $\pi_{\mu}$ we would have $h\left(\pi_{\mu}, I(\mu)\right)=h\left(\pi_{0}, I(0)\right)=h\left(\pi_{0},\{0\} \times H(f) \times H(g)\right) \neq \overline{0}$ and thus $I(\mu) \neq \varnothing$ for $\mu \in[0,1]$. This in turn would imply the existence of a bounded solution to $(2.1)^{*}$ for some $\left(f^{*}, g^{*}\right) \in H(f) \times H(g)$. In order to show the existence of such a compact isolating neighborhood $N$ it suffices to show that there is an a priori bound on the norms of all possible bounded solutions to

$$
x^{\prime}=f^{*}(x, \mu t)+\mu g^{*}(x, \mu t)
$$

The bound must be independent of $\mu \in[0,1]$ and $\left(f^{*}, g^{*}\right) \in H(f) \times H(g)$.

Suppose there are sequences $\left\{\left(f_{n}, g_{n}\right)\right\} \subset H(f) \times H(g),\left\{\mu_{n}\right\} \subset[0,1]$, and $\left\{x_{n}\right\} \subset C^{1}\left(\mathbb{R}, \mathbb{R}^{N}\right)$ such that each $x_{n}(t)$ is bounded on $\mathbb{R}$ with

$$
\sup _{t \in \mathbb{R}}\left|x_{n}(t)\right|=\left\|x_{n}\right\| \rightarrow \infty
$$

as $n \rightarrow \infty$, and

$$
x_{n}^{\prime}=f_{n}\left(x_{n}, \mu_{n} t\right)+\mu_{n} g\left(x_{n}, \mu_{n} t\right)
$$

for $n \in \mathbb{N}$ and $t \in \mathbb{R}$.

We may choose $\tau_{n} \in \mathbb{R}$ so that $\left|x_{n}\left(\tau_{n}\right)\right| \geq \frac{1}{2}\left\|x_{n}\right\|$. Let $y_{n}(t)$ be defined by

$$
y_{n}(t)=x_{n}\left(t\left\|x_{n}\right\|^{1-p}+\tau_{n}\right) /\left\|x_{n}\right\| \text {. }
$$

We have $\left\|y_{n}\right\|=1$ and $\left|y_{n}(0)\right| \geq \frac{1}{2}$ for $n \in \mathbb{N}$. By using the $p$-homogeneity of $f_{n}, n \in \mathbb{N}$, we get

$$
y_{n}^{\prime}=f_{n}\left(y_{n}, t\left\|x_{n}\right\|^{1-p}+\tau_{n}\right)+\tilde{g}_{n}(t)
$$

where

$$
\tilde{g}_{n}(t)=g_{n}\left(x_{n}\left(t\left\|x_{n}\right\|^{1-p}+\tau_{n}\right), t\left\|x_{n}\right\|^{1-p}+\tau_{n}\right) /\left\|x_{n}\right\|^{p} .
$$

There is thus a constant $C>0$ such that $\left\|y_{n}^{\prime}\right\|<C$ for $n \in \mathbb{N}$ and we may assume without loss of generality that $\left\{y_{n}\right\}$ converges in $C_{c}\left(\mathbb{R}, \mathbb{R}^{N}\right)$ to some bounded function $y$. Since $\left|y_{n}(0)\right| \geq \frac{1}{2}$ for all $n$, we have $y \neq 0$. Since $H(f)$ is compact in $C_{c}$ there is also no loss of generality in assuming $f_{n}\left(x, t+\tau_{n}\right)$ converges to some $f^{*}(x, t)$ in $C_{c}$. By (H4) $\tilde{g}_{n} \rightarrow 0$ as $n \rightarrow \infty$ and we see that $y=y(t)$ solves $y^{\prime}=f^{*}(y, 0)$. Since $y \neq 0$ is bounded on $\mathbb{R}$, this contradicts (H2).

Thus the a priori bound is established, and our arguments at the beginning of this step show there is some $\left(f^{*}, g^{*}\right) \in H(f) \times H(g)$ for which $(2.1)^{*}$ has a bounded solution. This proves the theorem.

Remark 2.1. Let $f^{*} \in H(f)$; since $f^{*}(x, 0)$ is homogeneous in $x \in \mathbb{R}^{N}$ it follows that (2.2) has no nonzero bounded solutions if and only if $\{0\}$ is an isolated invariant set for (2.2). Thus (H2) is really only a local assumption for each $f^{*}$. If $f(x, t)=\frac{\partial F}{\partial x}(x, t)$ for some $C^{1}$ function $F$ then under reasonable hypotheses each $f^{*}(x, 0)$ is a gradient function; in this case $(\mathrm{H} 2)$ holds if $x=0$ is, for each $f^{*}$, an isolated equilibrium point. Of course, if $f$ is independent of $t$ then $(\mathrm{H} 2)$ may be easy to check. Other examples are given in the last section. 
Remark 2.2. Instead of the flow $\beta$ consider the flow $\gamma$ on $\mathbb{R}^{N} \times H(f, g)$ defined by $\gamma\left(v,\left(f^{*}, g^{*}\right), t\right)=\left(\beta\left(v, f^{*}, t\right), f^{*}, g^{*}\right)$ for $\left(f^{*}, g^{*}\right) \in H(f, g)$. If

$$
h_{2}=h(\gamma,\{0\} \times H(f, g)) \neq \overline{0},
$$

virtually the same proof implies that $(2.1)^{*}$ has a bounded solution for some $\left(f^{*}, g^{*}\right) \in H(f, g)$. However the calculation of $h_{2}$ sometimes is more difficult than $h_{1}$.

If $h_{2} \neq \overline{0}$ and $f, g$ are each uniformly almost periodic in $t$ then one can show that the original differential equation $(2.1)$ has a bounded solution. The argument for this is a standard one: By $h_{2} \neq \overline{0}$ one has a bounded solution $u=u(t)$ of $(2.1)^{*}$ for some $\left(f^{*}, g^{*}\right) \in H(f, g)$. Now $t \mapsto(f(x, t), g(x, t))$ is uniformly almost periodic, so $(f, g) \in H\left(f^{*}, g^{*}\right)$ (see [F]). Hence there is a sequence $\left(\tau_{n}\right) \in \mathbb{R}$ such that $f_{\tau_{n}}^{*} \rightarrow f$ and $g_{\tau_{n}}^{*} \rightarrow g$ as $n \rightarrow \infty$. Let $u_{n}(t)=u\left(t+\tau_{n}\right)$; then $u_{n}$ satisfies

$$
u_{n}^{\prime}=f_{\tau_{n}}^{*}\left(u_{n}(t), t\right)+g_{\tau_{n}}^{*}\left(u_{n}(t), t\right) .
$$

We have $\left|u_{n}(t)\right|$ and hence $\left|u_{n}^{\prime}(t)\right|$ bounded on $\mathbb{R}$. A subsequence of $\left(u_{n}\right)$ converges uniformly on compact sets to a bounded function $y$. It is now easy to show that

$$
y^{\prime}(t)=f(y(t), t)+g(y(t), t) .
$$

Remark 2.3. In view of Remark 2.2 we will later make use of conditions which imply $H(f, g)=H(f) \times H(g)$ for uniformly almost periodic $f$ and $g$. In this case $h_{1} \neq \overline{0}$ implies $h_{2} \neq \overline{0}$ and we can apply Remark 2.1 to get bounded solutions of equation (2.1). In particular, if $f(x, t)$ is $T$-periodic in $t, g(x, t)$ is $\omega$-periodic in $T$ and $T / \omega$ is irrational then it is easy to show $H(f, g)=$ $H(f) \times H(G)$.

Remark 2.4. If each of the hulls $H(f)$ and $H(g)$ are equivalent homotopically to $H(f, g)$ then the method of Remark 2.2 is preferable. For example, if each of $f(x, t)$ and $g(x, t)$ and $T$-periodic in $t$ then each of $H(f), H(g)$, and $H(f, g)$ are topologically equivalent to $S^{1}$, whereas $H(f) \times H(g)$ is a torus.

In this case we state the following theorem.

Theorem 2.2. Let $f$ and $g$ be as in Theorem 2.1, and also suppose there exists $T>0$ such that $f(x, t+T)=f(x, t)$ and $g(x, t+T)=g(x, t)$ for all $(x, t) \in \mathbb{R}^{N} \times \mathbb{R}$. Again assume $(\mathrm{H} 1)-(\mathrm{H} 4)$ of Theorem 2.1 .

Then if $h_{1} \neq \overline{0}$ the original equation (2.1) has a bounded solution.

Remark 2.5. Notice that here $H(f, g) \neq H(f) \times H(g)$ so that this situation is complementary to the one discussed in Remark 2.3.

Proof. The proof is like that of Theorem 2.1, but using Remark 2.2. Notice that since $H(f)=H(g)=H(f, g)=S^{1}$ the index $h_{2}$ is the same as the index $h_{1}$.

Remark 2.6. In case $f$ and $g$ are uniformly almost periodic in $t$ and $h_{2} \neq$ $\overline{0}$ we can conclude that $(2.1)^{*}$ has a bounded solution for each $\left(f^{*}, g^{*}\right) \in$ $H(f, g)$. If there is a compact set $K$ in $\mathbb{R}^{N}$ such that $(2.1)^{*}$ has, for each $\left(f^{*}, g^{*}\right) \in H(f, g)$, a unique solution with values in $K$ then these solutions must all be almost periodic; this is due to Amerio. Given in [F] are other interesting conditions which also imply almost periodicity of the solutions. 
Remark 2.7. Suppose (for simplicity) that $g=0$ in (2.1) and that for each $f^{*} \in H(f)$ the zero solution of $x^{\prime}=f^{*}(x, 0)$ is asymptotically stable. In this case $\{0\} \times H(f)$ is asymptotically stable for the flow $\beta$ and the index $h_{1}$ is a disconnected homotopy type. By $(\mathrm{H} 2) u=0$ is the only bounded solution to (2.1). It follows [W, Corollary 2.8] that $u=0$ is a uniformly asymptotically stable solution of (2.1). This contrasts sharply with the linear case; there are well-known examples of real square periodic matrix functions $A(t)$ such that freezing coefficients in $x^{\prime}=A(t) x$ produces asymptotically stable systems but the original system is unstable (and has only zero for a bounded solution).

Remark 2.8. More generally than in the preceding remark we may have $g \neq 0$. Suppose $h_{1}$ is not a connected homotopy type then if $S=I(1)=$ the largest bounded invariant set in $\mathbb{R}^{N} \times H(f) \times H(g)$ for the flow $\pi_{1}$ then $h\left(\pi_{1}, S\right)=$ $h_{1} \wedge\left[H(g)^{*}, z\right]$. This latter index must also be disconnected since both $h_{1}$ and $\left[H(g)^{*}, z\right]$ are. If $S$ itself is connected topologically then $S$ is asymptotically stable. More precisely, by Theorem 2.7 of [W], for each open neighborhood $U$ of $S$ there is an open neighborhood $V$ of $S$ such that if $\left(v, f^{*}, g^{*}\right) \in V$ then $\pi_{1}\left(v, f^{*}, g^{*}, t\right) \in U$ for all $t \geq 0$. Moreover if $d$ is the metric on $\mathbb{R}^{N} \times C_{c}$ then $d\left(\pi_{1}\left(v, f^{*}, g^{*}, t\right), S\right) \rightarrow 0$ as $t \rightarrow \infty$.

Remark 2.9. Theorems 2.1 and 2.2 and Remarks $2.1-2.8$ can be extended to higher order systems of the form

$$
x^{(n)}=f(x, t)+g(x, t), \quad x \in \mathbb{R}^{N} .
$$

The underlying phase space must now be $R^{q} \times H(f) \times H(g)$ with $q=n \cdot N$, of course. The proof is about the same as that for Theorem 2.1, making use of the fact that if $x(t)$ and $x^{(n)}(t)$ are bounded on $\mathbb{R}$ then so are $x^{(k)}(t)$, $1 \leq k \leq n-1$.

\section{LINEARIZATION AND MULTIPLICITY}

Let us first consider in this section perturbed linear problems such as

$$
x^{\prime}=A(t) x+G(x, t),
$$

where $x \in \mathbb{R}^{N}, A(t)$ is a continuous real $N \times N$ matrix, and $G: \mathbb{R}^{N} \times \mathbb{R} \rightarrow \mathbb{R}^{N}$ is continuous. We assume $A$ and $G$ are uniformly continuous and bounded on all sets of the form $K \times \mathbb{R}, K$ compact in $\mathbb{R}^{N}$, and $G$ is locally Lipschitz in its first variable, uniformly with respect to its second variable. We suppose also that

$$
\lim _{|x| \rightarrow 0} G(x, t) /|x|=0
$$

uniformly for $t \in \mathbb{R}$.

Consider the (local) flow $\pi_{1}$ on $\mathbb{R}^{N} \times H(A) \times H(G)$ given by

$$
\pi_{1}\left(v, A^{*}, G^{*}, t\right)=\left(x\left(t ; v, A^{*}, G^{*}\right), A_{t}^{*}, G_{t}^{*}\right)
$$

where $x\left(t ; v, A^{*}, g^{*}\right)$ is the solution to

$$
x^{\prime}=A^{*}(t) x+G^{*}(x, t), \quad x(0)=v .
$$

We wish to relate $\pi_{1}$ to the flow $\tilde{\pi}\left(v, A^{*}, t\right)=\left(x\left(t ; v, A^{*}\right), A_{t}^{*}\right)$ defined by

$$
x^{\prime}=A^{*}(t) x, \quad x(0)=v .
$$


Lemma 3.1. Suppose $x=0$ is the only bounded solution to (3.4) for each $A^{*} \in$ $H(A)$. Then $S=\{0\} \times H(A) \times H(G)$ is a compact isolated invariant set in the flow $\pi_{1}$ and $h\left(\pi_{1}, S\right)=h(\tilde{\pi},\{0\} \times H(A)) \wedge\left[H(G)^{*}, z\right]$.

Proof. For $\mu \in[0,1]$ and $\left(A^{*}, G^{*}\right) \in H(A) \times H(G)$ we consider the initial value problems

$$
\begin{gathered}
x^{\prime}=A^{*}(t) x+\mu G^{*}(x, t), \\
x(0)=v .
\end{gathered}
$$

The flows $\pi_{\mu}\left(v, A^{*}, G^{*}, t\right)=\left(x\left(t ; v, \mu, A^{*}, G^{*}\right), A_{t}^{*}, G_{t}^{*}\right)$ defined by (3.5), (3.6) are on $\mathbb{R}^{N} \times H(A) \times H(G)$, for $0 \leq \mu \leq 1$. Of course only local existence is assumed.

If $\{0\} \times H(A) \times H(G)$ is not an isolated invariant set for each $\pi_{\mu}$ with a common isolating neighborhood then there are for each integer $n \geq 1$, $\left(A_{n}, G_{n}\right) \in H(A) \times H(G), \mu_{n} \in[0,1]$, and a function $x_{n}$ on $\mathbb{R}$ such that

$$
x_{n}^{\prime}=A_{n}(t) x_{n}+\mu_{n} G_{n}\left(x_{n}, t\right)
$$

for all $t \in \mathbb{R}$ and $\left\|x_{n}\right\| \leq \frac{2}{n}$ and $\left|x\left(\tau_{n}\right)\right|=\frac{1}{n}$ for some $\tau_{n} \in \mathbb{R}$.

Letting $y_{n}(t)=x_{n}\left(t+\tau_{n}\right) /\left\|x_{n}\right\|$ we see that

$$
y_{n}^{\prime}=A_{n}\left(t+\tau_{n}\right) y_{n}+\mu_{n} \widetilde{G}_{n}(t)
$$

where $\widetilde{G}_{n}(t)=G_{n}\left(x_{n}\left(t+\tau_{n}\right), t+\tau_{n}\right) /\left\|x_{n}\right\|$.

Letting $n \rightarrow \infty$ and going to subsequences if necessary we obtain a bounded function $y \neq 0$ which solves $y^{\prime}=A^{*}(t) y$ for some $A^{*} \in H(A)$. This contradicts our hypothesis on $A$. Since there is a common (small) isolating neighborhood of $\{0\} \times H(A) \times H(G)$ we may perform the homotopy to the flow $\pi_{0}=\tilde{\pi} \times \pi$ where $\pi\left(G^{*}, t\right)=G_{t}^{*}$, and the result follows by taking the smash product of the indices.

Remark 3.1. The comments made in Remarks 2.2, 2.3, 2.4 may be adapted here. That is, we may consider flows on $\mathbb{R}^{N} \times H(A, G)$ instead of on $\mathbb{R}^{N} \times$ $H(A) \times H(G)$. Again, as in Remarks 2.3 and 2.4 this may be especially useful if either $H(A, G)=H(A)=H(G)$ or $H(A, G)=H(A) \times H(G)$ (here equal means homotopically equivalent). This is illustrated in the following result on nontrivial solutions.

The ideas behind Theorem 2.1 and Lemma 3.1 enable us to derive a condition for a nontrivial bounded solution to (2.1).

Theorem 3.2. Suppose $f$ and $g$ are T-periodic in $t$, locally Lipschitz in $x$, uniformly in $t$, satisfy $(\mathrm{H} 1)-(\mathrm{H} 4)$ of Theorem $2.1, g(0, t)=0$, for all $t \in \mathbb{R}$, and:

(C.1) If $A(t)=\frac{\partial g}{\partial x}(0, t)$ then for each $A^{*} \in H(A)$, zero is the only bounded solution of (3.4).

(C.2) Let $\theta(x, t)=g(x, t)-A(t) x$; let $\tilde{\pi}$ be the flow defined by (3.4) and let $h_{1}$ be as in Theorem 2.1. Suppose

$$
h\left(\pi_{0},\{0\} \times H(A)\right) \wedge\left[H(\theta, f)^{*}, z\right] \neq h_{1} \wedge\left[H(A, \theta, f)^{*}, z_{1}\right] .
$$

Then (2.1) has a nontrivial bounded solution.

Remark 3.2. Notice each of $H(A), H(\theta, f)$, and $H(A, \theta, f)$ is either a point or $S^{1}$. 
Proof. We consider a skew product flow determined by (2.1). For $\left(A^{*}, \theta^{*}, f^{*}\right)$ $\in H(A, \theta, f)$ let $\pi_{1}\left(v, A^{*}, \theta^{*}, f^{*}, t\right)=\left(x\left(t ; v, A^{*}, \theta^{*}, f^{*}\right), A_{t}^{*}, \theta_{t}^{*}, f_{t}^{*}\right)$ where $x$ is the solution to

$$
\begin{gathered}
x^{\prime}=A^{*}(t) x+\theta^{*}(x, t)+f^{*}(x, t) \\
x(0)=v .
\end{gathered}
$$

We use two homotopies essentially defined for $0 \leq \mu \leq 1$ by

$$
x^{\prime}=\mu A^{*}(\mu t) x+\mu \theta^{*}(x, \mu t)+f^{*}(x, \mu t),
$$

as in Theorem 2.1, and

$$
x^{\prime}=A^{*}(t) x+\mu \theta^{*}(x, t)+\mu f^{*}(x, t),
$$

as in Lemma 3.1.

In a small neighborhood of $S_{0}=\{0\} \times H(A, \theta, f),(3.10)$ and arguments like those of Lemma 3.1 show that the index of $S_{0}$ is $h\left(\pi_{0},\{0\} \times H(A)\right) \wedge$ $[H(\theta, f), z]$. On the other hand, (3.9) and arguments similar to those of Theorem 2.1 show that there is a largest compact invariant set $S_{1}$ for the flow $\pi_{1}$ and its index is $h_{1} \wedge\left[H(A, \theta, f), z_{1}\right]$. Since $h\left(\pi_{1}, S_{0}\right) \neq h\left(\pi_{1}, S_{1}\right)$ and $S_{0} \subset S_{1}$ we conclude that $(2.1)^{*}$ must have at least one nontrivial bounded solution $y$ for some $\left(A^{*}, \theta^{*}, f^{*}\right) \in H(A, \theta, f)$. But then there is a $\tau \in$ $\mathbb{R}$ such that $\left(A^{*}, \theta^{*}, f^{*}\right)=\left(A_{\tau}, \theta_{\tau}, f_{\tau}\right)$ and $u(t)=y(t-\tau)$ is a nontrivial bounded solution of (2.1).

Remark 3.3. If only almost periodicity is assumed we must seek a solution of (2.1) as the limit of $u_{n}(t)=y\left(t-\tau_{n}\right)$, as in Remark 2.3. But the limit function may be the zero solution.

Remark 3.4. If no characteristic multiplier of (3.4) is in modulus equal to 1 then (C.1) holds.

\section{A FEW SIMPLE EXAMPLES}

The conditions of Theorems 2.1 and 3.2 can in some interesting cases be easily checked. Our examples are chosen to illustrate this.

Example 1. Let $p_{i}(t), i=1,2,3$, be continuous, positive, $T$-periodic functions. Consider the system

$$
x^{\prime}=y-p_{1}(t) x^{3}, \quad y^{\prime}=z+p_{2}(t) y^{3}, \quad z^{\prime}=x+p_{3}(t) z^{3} .
$$

First of all, $h_{1}=\Sigma^{2} \wedge\left[S^{1}, z\right]$ (cf. [C]); here $\left[S^{1}, z\right]=\left[S^{1} \bigcup^{\prime}\{z\}, z\right]$. In notation of Theorem 3.2, $A$ is a constant matrix, $\theta=0$, and $f$ is $T$-periodic. Thus

$$
\left[H(A, \theta, f)^{*}, z_{1}\right]=\left[H(f)^{*}, z_{1}\right]=\left[S^{1}, z_{1}\right], \quad z \notin S^{1},
$$

and

$$
\left[H(\theta, f)^{*}, z\right]=\left[S^{1}, z\right], \quad z \notin S^{1} .
$$

Now $A$ has one eigenvalue with positive real part and two eigenvalues with negative real part. Thus $h\left(\pi_{0},\{0\} \times H(A)\right)=\Sigma^{1}$. We have

$$
\left.h\left(\pi_{0},\{0\}\right) \times H(A)\right) \wedge\left[H(\theta, f)^{*}, z\right]=\Sigma^{1} \wedge\left[S^{1}, z\right], \quad z \notin S^{1},
$$


and

$$
h_{1} \wedge\left[H(A, \theta, f)^{*}, z_{1}\right]=\Sigma^{2} \wedge\left[S^{1}, z\right] \wedge\left[S^{1}, z\right], \quad z \notin S^{1} .
$$

It is easy to show [C] that these two indices are different. Thus Theorem 3.2 implies that (4.1) has a nontrivial bounded solution.

Example 2. Let $p: \mathbb{R} \rightarrow \mathbb{R}^{2}$ be a continuous periodic function of period $T>0$ where $\pi / T$ is irrational. Consider

$$
\left(\begin{array}{l}
x^{\prime} \\
y^{\prime}
\end{array}\right)=\left(\begin{array}{cc}
\sin t & \cos t \\
\cos t & -\sin t
\end{array}\right)\left(\begin{array}{l}
x^{3} \\
y^{3}
\end{array}\right)+p(t) .
$$

Fix $\tau \in[0,2 \pi]$ and consider

$$
x^{\prime}=\sin (\tau) x^{3}+\cos (\tau) y^{3}, \quad y^{\prime}=\cos (\tau) x^{3}-\sin (\tau) y^{3} .
$$

Let $g(x, y)=\frac{1}{4} x^{4}-\frac{1}{4} y^{4}$. Then along solutions

$$
\frac{d}{d t} g(x, y)=\sin (\tau)\left(x^{6}+y^{6}\right) .
$$

Thus for $\tau \neq 0, \pi$ the system (4.3) is gradient-like [C, p. 13] and can have only the isolated equilibrium $(0,0)$ as a bounded solution. When $\tau=0$ or $\pi$ this may be verified directly. Thus $(0,0)$ is an isolated invariant set for all values of $\tau$ and the homotopy index is a constant, say $h_{0}$. The case $\tau=0$ is easily shown to have index $\Sigma^{1}$. Thus $h_{0}=\Sigma^{1} \neq \overline{0}$. Unlike the previous example, the flow $\beta$ for this system is not a product. Viewing (4.3) as a family of systems parameterized by $\tau$ in $\mathbb{R}^{2} \times[0,2 \pi]$, one sees that as $\tau$ changes a rotation in the $x, y$ axes results. As $\tau$ varies from 0 to $2 \pi$, the axes rotate through an angle of $\pi$ radians. The exit sets from a neighborhood of the origin undergo the same rotation. Identifying the end points $\tau=0$ and $\tau=2 \pi$ one sees that the union of exit sets, $0 \leq \tau<2 \pi$, corresponds to the edge of a Moebius strip. Identification of the exit sets gives the index $h(\beta,\{0\} \times H(f))$ : it is the pointed two-dimensional projective space [C, p. 11]. By Theorem 2.1 and Remark 2.3, equation (4.2) has a bounded solution.

Remark 4.1. More generally in Example 2 one may assume that the module $\operatorname{Mod}(p)$ and $\operatorname{Mod}(A)$ has intersection $\{0\}$ (where $A$ is the sin, cos matrix in (4.2). That is, $Z \cap \operatorname{Mod}(p)=\{0\}$. The details will be omitted here. In case $p$ is $2 \pi$-periodic, it is already known that $(4.2)$ has a $2 \pi$-periodic solution $[\mathrm{K}-\mathrm{Z}$, p. 249].

\section{REFERENCES}

[C] C. Conley, Isolated invariant sets and the Morse index, CBMS Regional Conf. Ser. in Math., no. 38, Amer. Math. Soc., Providence, R.I., 1978.

[F] A. M. Fink, Almost periodic differential equations, Lecture Notes in Math., vol. 377, Springer-Verlag, Berlin, 1974.

[K-Z] M. A. Krasnosel'skii and P. P. Zabreiko, Geometrical methods of nonlinear analysis, Grundlehren der Math. Wissenschaften, 263, Springer-Verlag, Berlin, 1984.

[M] E. Muhamadiev, On the theory of periodic solutions of systems of ordinary differential equations, Dokl. Akad. Nauk SSSR (N.S.) 194 (1970), 510-513; English transl., Soviet Math. Dokl. 11 (1970), 1236-1239.

[Me-W] M. Memory and J. R. Ward, Jr., Conley index and the method of averaging, J. Math. Anal. Appl. 158 (1991), 509-518. 
[R] K. P. Rybakowski, The homotopy index and partial differential equations, Springer-Verlag, Berlin, 1987.

[Se] G. R. Sell, Topological dynamics and ordinary differential equations, Van Nostrand Rheinhold, London, 1971.

[Sm] J. Smoller, Shock waves and reaction-diffusion equations, Grundlehren der Math. Wissenschaften, 248, Springer-Verlag, New York, 1983.

[W] J. R. Ward, Jr., Conley index and non-autonomous ordinary differential equations, Results in Math. 14 (1988), 191-209.

[W.1] _ Averaging, homotopy, and bounded solutions of ordinary differential equations, Differential and Integral Equations 3 (1990), 1093-1100.

Department of Mathematics, University of Alabama at Birmingham, Birmingham, AlABAMA 35294 